\title{
Preface: Biofluid mechanics
}

\author{
James B. Grotberg \\ Biomedical Engineering Department, The University of Michigan, Ann Arbor, Michigan 48109-2099
}

(Received 7 January 2005; accepted 9 January 2005; published online 22 February 2005)

[DOI: $10.1063 / 1.1862617$ ]

Why biofluid mechanics? It is a fair question since Physics of Fluids embarked on this special section of papers dedicated to the subject. After some thought, my answer is the relatively simple view that life is a wet and sticky business, to be sure. And to our knowledge it is mostly immersed in our earthly atmosphere. So as long as people and other organisms strive to live, reproduce, and interact with who and what is around them, biofluid mechanics will have a steady stream of challenges. The vast scope of fluid physics, from nano/micro to galactic scales, is certainly understood by the audience of this journal. Biofluid mechanics, while not stretched to such a range of characteristic lengths, is equally vast in its richness of complex fluid properties and unique boundary conditions. For example, phonation derives from the interplay of airflow through the vocal cords, the results varying from love songs to crying to lectures in introductory fluid mechanics. The coupling of air vibrations to the cochlear apparatus of the ear gives the speaker/singer an audience. And it is the homeostatic balance of fluid flow in the eye that lets the reader read. Without healthy biofluid mechanics, we are all potentially deaf, dumb, and blind. By the way, biofluid mechanics researchers are busy in developing diagnostic and assistive modalities for those who do face such obstacles in their daily life.

The field of biofluid mechanics is a growing area of research and Physics of Fluids welcomes original contributions. One clear indicator of the increasing interest and activity in this field is the rapid expansion of presentations witnessed at the 57th Annual Meeting of the Division of Fluid Dynamics during 21-23 November 2004 in Seattle, Washington. There were 10 sessions entitled Bio-Fluid Dy- namics I-X over the three-day conference period. In addition to the 94 talks in those sessions, there were many others involving biological flows scattered elsewhere in the program. The list of treated topics is quite broad, including flows related to: aneurysms, stenotic blood vessels, stents, flapping flight, experimental methods, dispersion, biomimetics, bioreactors, microfluidics, microcirculation, lab-on-chip devices, animal-on-chip devices, large blood vessels, heart valves, fluid-elastic interactions, non-Newtonian flows, respiration, vocalization, artificial lungs, swimming by animals and actuated models, walking on water with feet or tails, collapsible tubes as conduits or as pumps, interactions with cells, and the mosquito proboscis. The range of applications was perhaps best seen in one of the sessions where "Measuring vortex breakdown flows within open cylindrical bioreactors using stereoscopic particle image velocimetry" was immediately followed by "Sniffers."

The collection of papers in this special section of Physics of Fluids is dedicated to biofluid mechanics and is representative of those seeking new fluid physics in the biological realm. From this interface, one hopes that there is both new fluid mechanics and new biology. These goals often go handin-hand. The papers cover interesting aspects of two-phase flow and stability in the lung where moving and elastic wall boundaries interact with surface tension and flow dynamics, cell-cell and cell-wall interactions in blood flow with complex cell and wall models to account for micro and molecular structure and function, and swimming of spirochetes. I wish to express my thanks to these authors for sharing this work with us. 